\title{
A HEAT CONDUCTION PROBLEM WITH SOURCES DEPENDING ON THE AVERAGE OF THE HEAT FLUX ON THE BOUNDARY
}

\author{
MAHDI BOUKROUCHE AND DOMINGO A. TARZIA
}

\begin{abstract}
Motivated by the modeling of temperature regulation in some mediums, we consider the non-classical heat conduction equation in the domain $D=\mathbb{R}^{n-1} \times \mathbb{R}^{+}$for which the internal energy supply depends on an average in the time variable of the heat flux $(y, s) \mapsto V(y, s)=u_{x}(0, y, s)$ on the boundary $S=\partial D$. The solution to the problem is found for an integral representation depending on the heat flux on $S$ which is an additional unknown of the considered problem. We obtain that the heat flux $V$ must satisfy a Volterra integral equation of the second kind in the time variable $t$ with a parameter in $\mathbb{R}^{n-1}$. Under some conditions on data, we show that a unique local solution exists, which can be extended globally in time. Finally in the one-dimensional case, we obtain the explicit solution by using the Laplace transform and the Adomian decomposition method.
\end{abstract}

\section{INTRODUCTION}

Let us consider the domain $D$ and its boundary $S$ defined by

$$
\begin{aligned}
& D=\mathbb{R}^{n-1} \times \mathbb{R}^{+}=\left\{(x, y) \in \mathbb{R}^{n}: x=x_{1}>0, y=\left(x_{2}, \ldots, x_{n}\right) \in \mathbb{R}^{n-1}\right\}, \\
& S=\partial D=\mathbb{R}^{n-1} \times\{0\}=\left\{(x, y) \in \mathbb{R}^{n}: x=0, y \in \mathbb{R}^{n-1}\right\} .
\end{aligned}
$$

The aim of this paper is to study the following Problem 1.1 on the non-classical heat equation, in the semi- $n$-dimensional space domain $D$ with nonlocal sources, for which the internal energy supply depends on the average $\frac{1}{t} \int_{0}^{t} u_{x}(0, y, s) d s$ of the heat flux on the boundary $S$.

2010 Mathematics Subject Classification. 35C15, 35K05, 35K20, 35K60, 45D05, 45E10, $80 \mathrm{~A} 20$.

Key words and phrases. Non-classical $n$-dimensional heat equation; Nonlocal sources; Volterra integral equation; Existence and uniqueness of solution; Integral representation of the solution; Explicit solution; Adomian decomposition method.

This paper was partially sponsored by the Institute Camille Jordan, Lyon University, UJM for the first author, by projects PIP \#0275 from CONICET - Universidad Austral and ANPCyT PICTO Austral 2016 \#090 (Rosario, Argentina) for the second author, and by the European Union's Horizon 2020 Research and Innovation Programme within the Marie Sklodowska-Curie grant agreement $823731 \mathrm{CONMECH}$. 
Problem 1.1. Find the temperature $u$ at $(x, y, t)$ satisfying the following conditions:

$$
\begin{aligned}
& u_{t}-\Delta u=-F\left(\frac{1}{t} \int_{0}^{t} u_{x}(0, y, s) d s\right), \quad x>0, y \in \mathbb{R}^{n-1}, t>0 \\
& u(0, y, t)=0, \quad y \in \mathbb{R}^{n-1}, t>0 \\
& u(x, y, 0)=h(x, y), \quad x>0, y \in \mathbb{R}^{n-1}
\end{aligned}
$$

where $\Delta$ denotes the Laplacian in $\mathbb{R}^{n}$.

This problem is motivated by modeling the temperature in an isotropic medium with the average of non-uniform and nonlocal sources that provide a cooling or heating system, according to the properties of the function $F$ with respect to the heat flow $(y, s) \mapsto V(y, s)=u_{x}(0, y, s)$ at the boundary $S$, see [11, 13. Some references on the subject are [6], where $F\left(\frac{1}{t} \int_{0}^{t} u_{x}(0, y, s) d s\right)$ is replaced by $F\left(u_{x}(0, y, t)\right)$, or [7], where it is replaced by $F\left(\int_{0}^{t} u_{x}(0, y, s) d s\right)$; see also [4, 14, 23, 24], where the semi-infinite case of this nonlinear problem with $F\left(u_{x}(0, y, t)\right)$ has been considered. The non-classical one-dimensional heat equation in a slab with fixed or moving boundaries was studied in [14, 22]. See also other references on the subject: [8]-[10], [12], [16]-[19]. To our knowledge, this is the first time that the solution to the average of a non-classical heat conduction of the type of Problem 1.1 is given. Other non-classical problems can be found in [5].

In [6] the basic solution to the $n$-dimensional heat equation and a technical lemma were established. We prove in Section 2 the local existence of a solution for the considered Problem 1.1 under some conditions on the data $F$ and $h$ which can be extended globally in time. Moreover, in Section 3 we consider the corresponding one dimensional problem and we obtain its explicit solution for the heat flux and the average of the total heat flux at the face $x=0$, by using the Laplace transform and also the Adomian decomposition method [1, 2, 3, 7, 25, 26].

\section{EXISTENCE RESUlts}

In this Section, we give first, in Theorem 2.1 the integral representation 2.2 of the solution of Problem 1.1, but it depends on the heat flow $V$ on the boundary $S$, which satisfies the Volterra integral equation (2.3) with initial condition (2.4). Then we prove, in Theorem 2.3 under some assumptions on the data, that there exists a unique solution of the problem locally in time which can be extended globally in time.

We first recall here the Green's function for the $n$-dimensional heat equation with homogeneous Dirichlet boundary conditions, given by the following expression:

$$
G_{1}(x, y, t ; \xi, \eta, \tau)=\frac{\exp \left[-\frac{\|y-\eta\|^{2}}{4(t-\tau)}\right]}{(2 \sqrt{\pi(t-\tau)})^{n-1}} G(x, t, \xi, \tau),
$$


where $G$ is the Green's function for the one-dimensional case given by

$$
G(x, t, \xi, \tau)=\frac{e^{-\frac{(x-\xi)^{2}}{4(t-\tau)}}-e^{-\frac{(x+\xi)^{2}}{4(t-\tau)}}}{2 \sqrt{\pi(t-\tau)}}, \quad t>\tau .
$$

Theorem 2.1. The integral representation of a solution of Problem 1.1 is given by the following expression:

$$
\begin{aligned}
& u(x, y, t)=u_{0}(x, y, t) \\
& -\int_{0}^{t} \frac{\operatorname{erf}\left(\frac{x}{2 \sqrt{t-\tau}}\right)}{(2 \sqrt{\pi(t-\tau)})^{n-1}}\left[\int_{\mathbb{R}^{n-1}} e^{\left[-\frac{\|y-\eta\|^{2}}{4(t-\tau)}\right]} F\left(\frac{1}{\tau} \int_{0}^{\tau} V(\eta, s) d s\right) d \eta\right] d \tau,
\end{aligned}
$$

where $\zeta \mapsto \operatorname{erf}(\zeta)=\left(\frac{2}{\sqrt{\pi}} \int_{0}^{\zeta} e^{-X^{2}} d X\right)$ is the error function,

$$
u_{0}(x, y, t)=\int_{D} G_{1}(x, y, t ; \xi, \eta, 0) h(\xi, \eta) d \xi d \eta
$$

and the heat flux $(y, t) \mapsto V(y, t)=u_{x}(0, y, t)$ on the surface $x=0$, satisfies the Volterra integral equation

$$
\begin{aligned}
& V(y, t)=V_{0}(y, t) \\
& -2 \int_{0}^{t} \frac{1}{(2 \sqrt{\pi(t-\tau)})^{n}}\left[\int_{\mathbb{R}^{n-1}} e^{\left[-\frac{\|y-\eta\|^{2}}{4(t-\tau)}\right]} F\left(\frac{1}{\tau} \int_{0}^{\tau} V(\eta, s) d s\right) d \eta\right] d \tau
\end{aligned}
$$

in the variable $t>0$, with $y \in \mathbb{R}^{n-1}$ a parameter, where

$$
V_{0}(y, t)=\int_{D} G_{1, x}(0, y, t ; \xi, \eta, 0) h(\xi, \eta) d \xi d \eta
$$

Proof. As the boundary condition in Problem 1.1 is homogeneous, we have from [15, 20]

$$
\begin{aligned}
u(x, y, t)= & \int_{D} G_{1}(x, y, t ; \xi, \eta, 0) h(\xi, \eta) d \xi d \eta \\
& -\int_{0}^{t} \int_{D} G_{1}(x, y, t ; \xi, \eta, \tau) F\left(\frac{1}{\tau} \int_{0}^{\tau} V(\eta, s) d s\right) d \xi d \eta d \tau
\end{aligned}
$$

and therefore

$$
\begin{aligned}
u_{x}(x, y, t)= & \int_{D} G_{1, x}(x, y, t ; \xi, \eta, 0) h(\xi, \eta) d \xi d \eta \\
& -\int_{0}^{t} \int_{D} G_{1, x}(x, y, t ; \xi, \eta, \tau) F\left(\frac{1}{\tau} \int_{0}^{\tau} V(\eta, s) d s\right) d \xi d \eta d \tau
\end{aligned}
$$


From (2.1) (the definition of $G_{1}$ ) by differentiation with respect to $x$, taking $x=0$ we obtain

$$
\begin{aligned}
\int_{D} G_{1, x} & (0, y, t ; \xi, \eta, \tau) F\left(\frac{1}{\tau} \int_{0}^{\tau} V(\eta, s) d s\right) d \xi d \eta \\
= & \int_{\mathbb{R}^{n-1}} \frac{F\left(\frac{1}{\tau} \int_{0}^{\tau} V(\eta, s) d s\right) e^{-\frac{\|y-\eta\|^{2}}{4(t-\tau)}}}{(t-\tau)^{\frac{n+2}{2}}(2 \sqrt{\pi})^{n}}\left(\int_{0}^{+\infty} \xi e^{-\frac{\xi^{2}}{4(t-\tau)}} d \xi\right) d \eta \\
= & \frac{2}{(2 \sqrt{\pi(t-\tau)})^{n}} \int_{\mathbb{R}^{n-1}} F\left(\frac{1}{\tau} \int_{0}^{\tau} V(\eta, s) d s\right) e^{-\frac{\|y-\eta\|^{2}}{4(t-\tau)}} d \eta
\end{aligned}
$$

Thus taking $x=0$ in 2.6 with 2.7 we get 2.3 .

Also by 2.1 we obtain

$$
\begin{aligned}
& \int_{D} G_{1}(x, y, t ; \xi, \eta, \tau) F\left(\frac{1}{\tau} \int_{0}^{\tau} V(\eta, s) d s\right) d \xi d \eta \\
& =\frac{1}{\left(2(\sqrt{\pi(t-\tau)})^{n}\right.} \int_{D} e^{\frac{-\|y-\eta\|^{2}}{4(t-\tau)}}\left[e^{-\frac{(x-\xi)^{2}}{4(t-\tau)}}-e^{-\frac{(x+\xi)^{2}}{W(t-\tau)}}\right] F\left(\frac{1}{\tau} \int_{0}^{\tau} V(\eta, s) d s\right) d \xi d \eta \\
& =\frac{1}{\left(2(\sqrt{\pi(t-\tau)})^{n}\right.} \int_{\mathbb{R}^{+}}\left[e^{-\frac{(x-\xi)^{2}}{4(t-\tau)}}-e^{-\frac{(x+\xi)^{2}}{4(t-\tau)}}\right] d \xi \\
& \times \int_{\mathbb{R}^{n-1}} e^{\frac{-\|y-\eta\|^{2}}{4(t-\tau)}} F\left(\frac{1}{\tau} \int_{0}^{\tau} V(\eta, s) d s\right) d \eta
\end{aligned}
$$

using

$$
\begin{aligned}
\int_{0}^{+\infty} e^{\frac{-(x-\xi)^{2}}{4(t-\tau)}} d \xi & =2 \sqrt{t-\tau}\left(\int_{-\infty}^{0} e^{-X^{2}} d X+\int_{0}^{\frac{x}{2 \sqrt{t-\tau}}} e^{-X^{2}} d X\right) \\
& =\sqrt{\pi(t-\tau)}\left(1+\operatorname{erf}\left(\frac{x}{2 \sqrt{t-\tau}}\right)\right)
\end{aligned}
$$

and

$$
\begin{aligned}
\int_{0}^{+\infty} e^{\frac{-(x+\xi)^{2}}{4(t-\tau)}} d \xi & =2 \sqrt{t-\tau}\left(\int_{0}^{+\infty} e^{-X^{2}} d X-\int_{0}^{\frac{x}{2 \sqrt{t-\tau}}} e^{-X^{2}} d X\right) \\
& =\sqrt{\pi(t-\tau)}\left(1-\operatorname{erf}\left(\frac{x}{2 \sqrt{t-\tau}}\right)\right)
\end{aligned}
$$

so we get

$$
\begin{aligned}
\int_{D} G_{1}(x, y, t ; \xi, \eta, \tau) F & \left(\frac{1}{\tau} \int_{0}^{\tau} V(\eta, s) d s\right) d \xi d \eta \\
= & \frac{\operatorname{erf}\left(\frac{x}{2 \sqrt{t-\tau}}\right)}{(2 \sqrt{\pi(t-\tau)})^{n-1}} \int_{\mathbb{R}^{n-1}} e^{-\frac{\|y-\eta\|^{2}}{4(t-\tau)}} F\left(\frac{1}{\tau} \int_{0}^{\tau} V(\eta, s) d s\right) d \eta .
\end{aligned}
$$

Taking this formula in 2.5 we obtain 2.2. 
Lemma 2.2. The simplified form of the Volterra integral equation (2.3) is given by

$$
\begin{aligned}
V(y, t)= & \frac{1}{t(2 \sqrt{\pi t})^{n}} \int_{\mathbb{R}^{+}} \xi e^{-\frac{\xi^{2}}{4 t}}\left(\int_{\mathbb{R}^{n-1}} e^{-\frac{\|y-\eta\|^{2}}{4 t}} h(\xi, \eta) d \eta\right) d \xi \\
& -\frac{2}{(2 \sqrt{\pi})^{n}} \int_{0}^{t} \frac{1}{(t-\tau)^{n / 2}} \int_{\mathbb{R}^{n-1}} F\left(\frac{1}{\tau} \int_{0}^{\tau} V(\eta, s) d s\right) e^{-\frac{\|y-\eta\|^{2}}{4(t-\tau)}} d \eta d \tau .
\end{aligned}
$$

Proof. Using the derivative with respect to $x$ of $(2.1$, then taking $x=0$ and $\tau=0$, and then taking the new expression of $V_{0}(y, t)$ in the Volterra integral equation 2.3, we obtain 2.8.

Theorem 2.3. Assume that $h \in \mathcal{C}(D), F \in \mathcal{C}(\mathbb{R})$ and locally Lipschitz in $\mathbb{R}$; then there exists a unique solution of Problem 1.1 locally in time which can be extended globally in time.

Proof. We know from Theorem 2.1 that, to prove the existence and uniqueness of the solution 2.2 of Problem 1.1 it is enough to solve the Volterra integral equation 2.8. So we rewrite it as

$$
V(y, t)=f(y, t)+\int_{0}^{t} g(y, \tau, V(y, \tau)) d \tau
$$

with

$$
f(y, t)=\frac{1}{t(2 \sqrt{\pi t})^{n}} \int_{\mathbb{R}^{+}} \xi e^{-\frac{\xi^{2}}{4 t}}\left(\int_{\mathbb{R}^{n-1}} e^{-\frac{\|y-\eta\|^{2}}{4 t}} h(\xi, \eta) d \eta\right) d \xi
$$

and

$$
g(t, \tau, y, V(y, \tau))=-\frac{2(t-\tau)^{-n / 2}}{(2 \sqrt{\pi})^{n}} \int_{\mathbb{R}^{n-1}} F\left(\frac{1}{\tau} \int_{0}^{\tau} V(\eta, s) d s\right) e^{-\frac{\|y-\eta\|^{2}}{4(t-\tau)}} d \eta .
$$

So we have to check the conditions $\mathrm{H} 1$ to $\mathrm{H} 4$ in [21, Theorem 1.1, p. 87], and H5 and H6 in [21, Theorem 1.2, p. 91].

- The function $f$ is defined and continuous for all $(y, t) \in \mathbb{R}^{n-1} \times \mathbb{R}^{+}$, so H1 holds.

- The function $g$ is measurable in $(t, \tau, y, x)$ for $0 \leq \tau \leq t<+\infty, x \in \mathbb{R}^{+}, y \in \mathbb{R}^{n-1}$, and continuous in $x$ for all $(y, t, \tau) \in \mathbb{R}^{n-1} \times \mathbb{R}^{+} \times \mathbb{R}^{+}, g(y, t, \tau, x)=0$ if $\tau>t$, so here we need the continuity of

$$
V(\eta, \tau) \mapsto F\left(\frac{1}{\tau} \int_{0}^{\tau} V(\eta, s) d s\right),
$$

which follows from the hypothesis that $F \in C(\mathbb{R})$. So H2 holds. 
- For all $k>0$ and any bounded set $B$ in $\mathbb{R}$, we have

$$
\begin{aligned}
|g(y, t, \tau, X)| & \leq \frac{2}{(2 \sqrt{\pi})^{n}} \sup _{X \in B}|F(X)|(t-\tau)^{-\frac{n}{2}} \int_{\mathbb{R}^{n-1}} e^{-\frac{\|y-\eta\|^{2}}{4(t-\tau)}} d \eta \\
& \leq \frac{2}{(2 \sqrt{\pi})^{n}} \sup _{X \in B}|F(X)|(t-\tau)^{-\frac{n}{2}}(2 \sqrt{\pi(t-\tau)})^{n-1} \\
& =\frac{1}{\sqrt{\pi}} \sup _{X \in B}|F(X)| \frac{1}{\sqrt{(t-\tau)}} ;
\end{aligned}
$$

thus there exists a measurable function $m$ given by

$$
m(t, \tau)=\frac{1}{\sqrt{\pi}} \sup _{X \in B}|F(X)| \frac{1}{\sqrt{(t-\tau)}}
$$

such that

$$
|g(y, t, \tau, X)| \leq m(t, \tau), \quad \forall 0 \leq \tau \leq t \leq k, X \in B,
$$

and

$$
\begin{aligned}
\sup _{t \in[0, k]} \int_{0}^{t} m(t, \tau) d \tau & =\frac{1}{\sqrt{\pi}} \sup _{X \in B}|F(X)| \sup _{t \in[0, k]} \int_{0}^{t} \frac{1}{\sqrt{t-\tau}} d \tau \\
& =\frac{1}{\sqrt{\pi}} \sup _{X \in B}|F(X)| \sup _{t \in[0, k]}\left(-\left.2 \sqrt{(t-\tau)}\right|_{0} ^{t}\right) \\
& =\frac{1}{\sqrt{\pi}} \sup _{X \in B}|F(X)| \sup _{t \in[0, k]} 2 \sqrt{t} \leq \frac{2 \sqrt{k}}{\sqrt{\pi}} \sup _{X \in B}|F(X)|<\infty,
\end{aligned}
$$

so H3 holds.

- Moreover, we also have

$$
\begin{aligned}
\lim _{t \rightarrow 0^{+}} \int_{0}^{t} m(t, \tau) d \tau & =\frac{1}{\sqrt{\pi}} \sup _{X \in B}|F(X)| \lim _{t \rightarrow 0^{+}} \int_{0}^{t} \frac{d \tau}{\sqrt{t-\tau}} \\
& =\frac{1}{\sqrt{\pi}} \sup _{X \in B}|F(X)| \lim _{t \rightarrow 0^{+}}(2 \sqrt{t})=0
\end{aligned}
$$

and

$$
\lim _{t \rightarrow 0^{+}} \int_{-T}^{-T+t} m(t, \tau) d \tau=\frac{1}{\sqrt{\pi}} \sup _{X \in B}|F(X)| \lim _{t \rightarrow 0^{+}} 2(\sqrt{t+T}-\sqrt{T})=0 .
$$

- For each compact subinterval $J$ of $\mathbb{R}^{+}$, each bounded set $B$ in $\mathbb{R}^{n-1}$, and each $t_{0} \in \mathbb{R}^{+}$, we set

$$
\mathcal{A}(t, y, V(\eta))=\left|g(t, \tau ; y, V(\eta, \tau))-g\left(t_{0}, \tau ; y, V(\eta, \tau)\right)\right| .
$$

By the definition of $g$ we get

$$
\begin{aligned}
& \mathcal{A}(t, y, V(\eta)) \\
& \quad=\frac{2}{(2 \sqrt{\pi})^{n}} \int_{J}\left|\int_{\mathbb{R}^{n-1}}\left[\frac{e^{-\frac{\|y-z\|^{2}}{4(t-\tau)}}}{(t-\tau)^{n / 2}}-\frac{e^{-\frac{\|y-z\|^{2}}{4\left(t_{0}-\tau\right)}}}{\left(t_{0}-\tau\right)^{n / 2}}\right] F\left(\frac{1}{\tau} \int_{0}^{\tau} V(z, s) d s\right) d z\right| d \tau .
\end{aligned}
$$


As the function $\tau \mapsto V(z, \tau)$ is continuous, then

$$
\tau \mapsto \frac{1}{\tau} \int_{0}^{\tau} V(z, s) d s
$$

is $\mathcal{C}^{1}\left(\mathbb{R}^{+}\right)$and is in the compact $B \subset \mathbb{R}$ for all $z \in \mathbb{R}^{n-1}$, so by the continuity of $F$ we get $F\left(\frac{1}{\tau} \int_{0}^{\tau} V(z, s) d s\right) \subset F(B)$, that is, there exists $M>0$ such that $\left|F\left(\frac{1}{\tau} \int_{0}^{\tau} V(z, s) d s\right)\right| \leq M$ for all $(z, \tau) \in \mathbb{R}^{n-1} \times \mathbb{R}^{+}$. So

$$
\mathcal{A}(t, y, V(\eta)) \leq \frac{2 M}{(2 \sqrt{\pi})^{n}}\left|\int_{\mathbb{R}^{n-1}} \frac{e^{-\frac{\|y-z\|^{2}}{4(t-\tau)}}}{\sqrt{(t-\tau)}^{n}} d z-\int_{\mathbb{R}^{n-1}} \frac{e^{-\frac{\|y-z\|^{2}}{4\left(t_{0}-\tau\right)}}}{\sqrt{\left(t_{0}-\tau\right)} n} d z\right|
$$

and by using

$$
\int_{\mathbb{R}^{n-1}} \exp \left[-\frac{\|y-z\|^{2}}{4(t-\tau)}\right] d z=(2 \sqrt{\pi(t-\tau)})^{n-1}
$$

we obtain

$$
\begin{aligned}
\mathcal{A}(t, y, V(\eta)) & \leq \frac{2 M}{(2 \sqrt{\pi})^{n}}\left|\frac{(2 \sqrt{\pi(t-\tau)})^{n-1}}{(\sqrt{t-\tau})^{n}}-\frac{\left(2 \sqrt{\pi\left(t_{0}-\tau\right)}\right)^{n-1}}{\left(\sqrt{t_{0}-\tau}\right)^{n}}\right| \\
& \leq \frac{M}{\sqrt{\pi}}\left|\frac{\sqrt{t_{0}-\tau}-\sqrt{t-\tau}}{\sqrt{(t-\tau)\left(t_{0}-\tau\right)}}\right| .
\end{aligned}
$$

Thus we deduce that

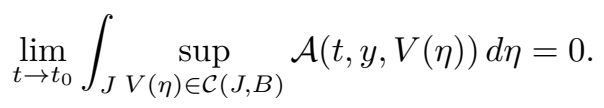

So H4 holds.

- For all compact $I \subset \mathbb{R}^{+}$, all $\psi \in \mathcal{C}\left(I, \mathbb{R}^{n}\right)$ and all $t_{0}>0$, we have:

$$
\begin{aligned}
& \left|g(t, \tau ; \psi(\tau))-g\left(t_{0}, \tau, \psi(\tau)\right)\right| \\
& \quad=\frac{2}{(2 \sqrt{\pi})^{n}}\left|\int_{\mathbb{R}^{n-1}} F\left(\frac{1}{\tau} \int_{0}^{\tau} \psi(s) d s\right)\left(\frac{e^{-\frac{\|y-\eta\|^{2}}{4(t-\tau)}}}{(t-\tau)^{n / 2}}-\frac{e^{-\frac{\|y-\eta\|^{2}}{4\left(t_{0}-\tau\right)}}}{\left(t_{0}-\tau\right)^{n / 2}}\right) d \eta\right| .
\end{aligned}
$$

As $F \in \mathcal{C}(\mathbb{R})$ and $\psi \in \mathcal{C}\left(I, \mathbb{R}^{n}\right)$, then there exists a constant $M>0$ such that

$$
\left|F\left(\frac{1}{\tau} \int_{0}^{\tau} \psi(s) d s\right)\right| \leq M, \quad \forall \tau \in I
$$

then we obtain, as for $\mathrm{H} 4$, that

$$
\lim _{t \rightarrow t_{0}} \int_{I}\left|g(t, \tau ; \psi(\tau))-g\left(t_{0}, \tau, \psi(\tau)\right)\right| d \tau=0
$$

So H5 holds.

- Now for each constant $k>0$ and each bounded set $B \subset \mathbb{R}^{n-1}$ there exists a measurable function $\varphi$ such that

$$
|g(y, t, \tau, x)-g(y, t, \tau, X)| \leq \varphi(t, \tau)|x-X|
$$


whenever $0 \leq \tau \leq t \leq k$ and both $x$ and $X$ are in $B$. Indeed, as $F$ is assumed to be a locally Lipschitz function in $\mathbb{R}$, there exists a constant $L>0$ such that

$$
\begin{aligned}
\left|F\left(\frac{1}{\tau} \int_{0}^{\tau} x(s) d s\right)-F\left(\frac{1}{\tau} \int_{0}^{\tau} X(s) d s\right)\right| & \leq L\left|\frac{1}{\tau} \int_{0}^{\tau} x(s) d s-\frac{1}{\tau} \int_{0}^{\tau} X(s) d s\right| \\
& \leq L|x-X|, \quad \forall(x, X) \in B^{2} ;
\end{aligned}
$$

then we have

$$
\begin{aligned}
|g(y, t, \tau, x)-g(y, t, \tau, X)| & \leq \frac{2 L}{(2 \sqrt{\pi})^{n}}\left(\int_{\mathbb{R}^{n-1}} e^{-\frac{\|y-\eta\|^{2}}{4(t-\tau)}} d \eta\right)(t-\tau)^{-n / 2}|x-X| \\
& \leq \frac{L}{\sqrt{\pi(t-\tau)}}|x-X|,
\end{aligned}
$$

and thus $\varphi(t, \tau)=\frac{L}{\sqrt{\pi(t-\tau)}}$. We have also that, for each $t \in[0, k]$, the function $\varphi$ is in $L^{1}(0, t)$ as a function of $\tau$, and we have also

$$
\int_{t}^{t+l} \varphi(t+l, \tau) d \tau=\frac{L}{\sqrt{\pi}} \int_{t}^{t+l} \frac{d \tau}{\sqrt{t+l-\tau}}=\frac{L}{\sqrt{\pi}}(2 \sqrt{l}) \rightarrow 0 \quad \text { with } l \rightarrow 0 .
$$

So H6 holds.

All the conditions $\mathrm{H} 1$ to $\mathrm{H} 6$ are satisfied with 2.9 and 2.10 .

Thus from [21, Theorems 1.1, 1.2 and 2.3] there exists a unique solution, local in time, to the Volterra integral equation 2.3 which can be extended globally in time. Then the proof of this theorem is complete.

\section{The one-Dimensional Case of Problem 1.1}

Let us consider now the one-dimensional case of Problem 1.1 for the temperature defined by

Problem 3.1. Find the temperature $u$ at $(x, t)$ such that it satisfies the following conditions:

$$
\begin{aligned}
u_{t}-u_{x x} & =-F\left(\frac{1}{t} \int_{0}^{t} u_{x}(0, s) d s\right), \quad x>0, t>0, \\
u(0, t) & =0, \quad t>0 \\
u(x, 0) & =h(x), \quad x>0 .
\end{aligned}
$$

Taking into account that

$$
\int_{0}^{t} G(x, t, \xi, \tau) d \xi=\operatorname{erf}\left(\frac{x}{2 \sqrt{t-\tau}}\right)
$$

the solution of Problem 3.1 is given by

$$
u(x, t)=u_{0}(x, t)-\int_{0}^{t} \operatorname{erf}\left(\frac{x}{2 \sqrt{t-\tau}}\right) F\left(\frac{1}{\tau} \int_{0}^{\tau} V(\sigma) d \sigma\right) d \tau
$$

with

$$
u_{0}(x, t)=\int_{0}^{t} G(x, t, \xi, 0) h(\xi) d \xi
$$


and $V(t)=u_{x}(0, t)$ the solution of the following Volterra integral equation of the second kind:

$$
V(t)=V_{0}(t)-\int_{0}^{t} \frac{F\left(\frac{1}{\tau} \int_{0}^{\tau} V(\sigma) d \sigma\right)}{\sqrt{\pi(t-\tau)}} d \tau
$$

where

$$
V_{0}(t)=\frac{1}{2 \sqrt{\pi} t^{3 / 2}} \int_{0}^{+\infty} \xi e^{-\xi^{2} / 4 t} h(\xi) d \xi=\frac{2}{\sqrt{\pi t}} \int_{0}^{+\infty} \eta e^{-\eta^{2}} h(2 \sqrt{t} \eta) d \eta .
$$

For the particular case

$$
h(x)=h_{0}>0, \text { for } x>0 \quad \text { and } \quad F(V)=\lambda V, \text { for } \lambda \in \mathbb{R}
$$

we have

$$
u_{0}(t, x)=h_{0} \operatorname{erf}\left(\frac{x}{2 \sqrt{t}}\right)
$$

and the integral equation $(3.1)$ becomes

$$
V(t)=\frac{h_{0}}{\sqrt{\pi t}}-\lambda \int_{0}^{t} \frac{\frac{1}{\tau} \int_{0}^{\tau} V(\sigma) d \sigma}{\sqrt{\pi(t-\tau)}} d \tau .
$$

Then, we have

$$
u(x, t)=h_{0} \operatorname{erf}\left(\frac{x}{2 \sqrt{t}}\right)-\lambda \int_{0}^{t} \operatorname{erf}\left(\frac{x}{2 \sqrt{t-\tau}}\right) W(\tau) d \tau,
$$

where $W(t)$ is defined by

$$
W(t)=\frac{1}{t} \int_{0}^{t} V(\tau) d \tau=\frac{1}{t} \int_{0}^{t} u_{x}(0, \tau) d \tau .
$$

By using the integral equation (3.3) for $V(t)$ we obtain for $W(t)$ the following Volterra integral equation of the second kind:

$$
\begin{aligned}
W(t) & =\frac{1}{t} \int_{0}^{t}\left[\frac{h_{0}}{\sqrt{\pi \tau}}-\lambda \int_{0}^{\tau} \frac{\frac{1}{\mu} \int_{0}^{\mu} V(\sigma) d \sigma}{\sqrt{\pi(\tau-\mu)}} d \mu\right] d \tau \\
& =\frac{1}{t}\left[2 h_{0} \sqrt{\frac{t}{\pi}}-\lambda \int_{0}^{t}\left[\int_{0}^{\tau} \frac{W(\mu)}{\sqrt{\pi(\tau-\mu)}} d \mu\right] d \tau\right] \\
& =\frac{2 h_{0}}{\sqrt{\pi t}}-\frac{\lambda}{t} \int_{0}^{t}\left[\int_{\mu}^{t} \frac{W(\mu)}{\sqrt{\pi(\tau-\mu)}} d \tau\right] d \mu \\
& =\frac{2 h_{0}}{\sqrt{\pi t}}-\frac{2 \lambda}{\sqrt{\pi}} \frac{1}{t} \int_{0}^{t} W(\tau) \sqrt{t-\tau} d \tau, \quad t>0,
\end{aligned}
$$

by using that

$$
\int_{\mu}^{t} \frac{d \tau}{\sqrt{\tau-\mu}}=2 \sqrt{t-\mu}
$$

Therefore, we deduce the following results. 
Theorem 3.1. Taking $h$ and $F$ as in (3.2), the solution of the non-classical heat conduction Problem 3.1 is given by (3.4), where $W(t)$ is the solution of the Volterra integral equation (3.6). Moreover, its Laplace transform $\mathcal{L}$ is given by the following expression:

$$
Q(s)=\mathcal{L}(W(t))(s)=\frac{h_{0}}{\lambda}\left(1-e^{-\frac{2 \lambda}{\sqrt{s}}}\right),
$$

and $W(t)$ is given by the following difference of two series with infinite radii of convergence:

$$
\begin{aligned}
W(t)= & \frac{2 h_{0}}{\sqrt{\pi t}}\left(1+\sum_{n=1}^{+\infty} \frac{\left(4 \lambda^{2} t\right)^{n}}{(2 n+1) n ![(2 n-1) ! !]^{2}}\right) \\
& -2 h_{0} \lambda\left(1+\sum_{n=1}^{+\infty} \frac{\left(2 \lambda^{2} t\right)^{n}}{(n+1)(n !)^{2}(2 n+1) ! !}\right) .
\end{aligned}
$$

Proof. By using the integral equation (3.6) for the real function $W(t)$, the Laplace transform $Q(s)$ of $W(t)$ satisfies the following first order ordinary differential problem:

$$
\begin{aligned}
& Q^{\prime}(s)-\frac{\lambda}{s^{3 / 2}} Q(s)=-\frac{h_{0}}{s^{3 / 2}}, \quad \Re(s)>0 \\
& Q(+\infty)=0,
\end{aligned}
$$

whose solution is given by (3.7). From a series development of the exponential function we obtain

$$
\begin{aligned}
Q(s) & =\frac{h_{0}}{\lambda} \sum_{n=1}^{+\infty} \frac{(-1)^{n+1}}{n !} \frac{(2 \lambda)^{n}}{s^{n / 2}} \\
& =\frac{h_{0}}{\lambda}\left(\sum_{k=0}^{+\infty} \frac{(2 \lambda)^{2 k+1}}{(2 k+1) ! s^{k+\frac{1}{2}}}-\sum_{k=1}^{+\infty} \frac{(2 \lambda)^{2 k}}{(2 k) ! s^{k}}\right) \\
& =2 h_{0}\left(\frac{1}{s^{\frac{1}{2}}}+\sum_{k=1}^{+\infty} \frac{(2 \lambda)^{2 k}}{(2 k+1)(2 k) ! s^{k+\frac{1}{2}}}-\sum_{k=1}^{+\infty} \frac{2^{k-1} \lambda^{2 k-1}}{k !(2 k-1) ! ! s^{k}}\right),
\end{aligned}
$$

and therefore we get

$$
\begin{aligned}
W(t)=\mathcal{L}^{-1}(Q(s))(t)= & 2 h_{0}\left(\frac{1}{\sqrt{\pi t}}+\frac{1}{\sqrt{\pi t}} \sum_{n=1}^{+\infty} \frac{(2 \lambda)^{2 n} 2^{n} t^{n}}{(2 n+1)(2 n) !(2 n-1) ! !}\right) \\
& -2 h_{0}\left(\lambda+\sum_{n=1}^{+\infty} \frac{2^{n} \lambda^{2 n+1} t^{n}}{(n+1) ! n !(2 n+1) ! !}\right),
\end{aligned}
$$

that is, the expression 3.8 for $W(t)$ holds by using that

$$
\begin{gathered}
\mathcal{L}^{-1}\left(\frac{1}{s^{\frac{1}{2}}}\right)(t)=\frac{1}{\sqrt{\pi t}}, \quad \mathcal{L}^{-1}\left(\frac{1}{s^{n}}\right)(t)=\frac{t^{n-1}}{(n-1) !}, \\
\mathcal{L}^{-1}\left(\frac{1}{s^{n+\frac{1}{2}}}\right)(t)=\frac{2^{n} t^{n-\frac{1}{2}}}{(2 n-1) ! ! \sqrt{\pi}}, \quad n \geq 1
\end{gathered}
$$




$$
(2 n) !=2^{n} n !(2 n-1) ! !
$$

and the definition

$$
(2 n-1) ! !=(2 n-1)(2 n-3) \cdots 5 \cdot 3 \cdot 1 .
$$

Corollary 3.2. The heat flux at the boundary $x=0$ of the solution of Problem 3.1 is given by

$$
u_{x}(0, t)=\frac{h_{0}}{\sqrt{\pi t}}-\frac{\lambda}{\sqrt{\pi}} \int_{0}^{t} \frac{W(\tau)}{\sqrt{t-\tau}} d \tau,
$$

where $W(t)$ is given by 3.8 .

Corollary 3.3. The first terms of the development of the series (3.8) of the average of the total heat flux at $x=0$ are given by

$$
2 h_{0}\left(\frac{1}{\sqrt{\pi t}}-\lambda+\frac{4 \lambda^{2}}{3 \sqrt{\pi}} \sqrt{t}-\frac{\lambda^{3}}{3} t+\frac{8 \lambda^{4}}{45 \sqrt{\pi}} t^{3 / 2}-\frac{\lambda^{5}}{45} t^{2}\right),
$$

which give us a singularity of $W$ of the type $t^{-1 / 2}$ at $t=0$.

Moreover, the first terms of the development of the heat flux at $x=0$ of the expression (3.9) are given by

$$
h_{0}\left(\frac{1}{\sqrt{\pi t}}-\frac{\lambda}{4}+\frac{4 \lambda^{2}}{\sqrt{\pi}} \sqrt{t}-\frac{4 \lambda^{3}}{3} t+\frac{8 \lambda^{4}}{9 \sqrt{\pi}} t^{3 / 2}-\frac{2 \lambda^{5}}{15} t^{2}+\frac{32 \lambda^{6}}{675 \sqrt{\pi}} t^{5 / 2}\right),
$$

which also give us a singularity of $u_{x}(0, t)$ of the type $t^{-1 / 2}$ at $t=0$.

Proof. It follows from the following results:

$$
\begin{aligned}
\int_{0}^{t} \frac{d \tau}{\sqrt{\tau(t-\tau)}}=\frac{\pi}{8}, & \int_{0}^{t} \frac{d \tau}{\sqrt{t-\tau}}=2 \sqrt{t}, \\
\int_{0}^{t} \frac{\sqrt{\tau}}{\sqrt{t-\tau}} d \tau=\frac{\pi}{2} t, & \int_{0}^{t} \frac{\tau}{\sqrt{t-\tau}} d \tau=\frac{4}{3} t^{3 / 2}, \\
\int_{0}^{t} \frac{\tau^{3 / 2}}{\sqrt{t-\tau}} d \tau=\frac{3 \pi}{8} t^{2}, & \int_{0}^{t} \frac{\tau^{2}}{\sqrt{t-\tau}} d \tau=\frac{16}{15} t^{5 / 2},
\end{aligned}
$$

which can be generalized to

$$
\begin{array}{ll}
\int_{0}^{t} \frac{\tau^{n}}{\sqrt{t-\tau}} d \tau=\frac{(2 n) !}{[(2 n-1) ! !]^{2}} \frac{t^{n+\frac{1}{2}}}{n+\frac{1}{2}}, \quad n \geq 1, \\
\int_{0}^{t} \frac{\tau^{n-\frac{1}{2}}}{\sqrt{t-\tau}} d \tau=\frac{\pi[(2 n-1) ! !]^{2}}{(2 n) !} t^{n}, \quad n \geq 1 .
\end{array}
$$

Now, we will give a new proof of the series $(3.8)$ for the average of the total flux $W(t)$. We use the Adomian decomposition method [1, 2, 3, 17, 25, 26] through a series expansion of the type

$$
W(t)=\sum_{n=0}^{+\infty} W_{n}(t)
$$


in the Volterra integral equation (3.6). Taking

$$
W_{0}(t)=\frac{2 h_{0}}{\sqrt{\pi t}}
$$

we obtain the recurrence formula

$$
W_{n}(t)=-\frac{2 \lambda}{t \sqrt{\pi}} \int_{0}^{t} W_{n-1}(\tau) \sqrt{t-\tau} d \tau, \quad n \geq 1 .
$$

Then by 3.10 and 3.11 we get

$$
\begin{aligned}
W_{1}(t) & =-\frac{2 \lambda}{t \sqrt{\pi}} \int_{0}^{t} W_{0}(\tau) \sqrt{t-\tau} d \tau \\
& =-\frac{4 \lambda h_{0}}{t \pi} \int_{0}^{t} \frac{\sqrt{t-\tau}}{\sqrt{\tau}} d \tau=-2 \lambda h_{0} .
\end{aligned}
$$

Theorem 3.4. By using a double induction principle we have

$$
W_{2 n}(t)=\frac{2 h_{0}}{\sqrt{\pi t}} \frac{\left(4 \lambda^{2} t\right)^{n}}{(2 n+1) n ![(2 n-1) ! !]^{2}}, \quad n \geq 1,
$$

and

$$
W_{2 n+1}(t)=-2 \lambda h_{0} \frac{\left(2 \lambda^{2} t\right)^{n}}{(n+1)(n !)^{2}(2 n+1) ! !}, \quad n \geq 1,
$$

with $W_{0}$ and $W_{1}$ given respectively by 3.10 and 3.12.

Proof. Using (3.10) and 3.11) we get

$$
\begin{aligned}
W_{2}(t) & =-\frac{2 \lambda}{t \sqrt{\pi}} \int_{0}^{t} W_{1}(\tau) \sqrt{t-\tau} d \tau \\
& =\frac{4 \lambda^{2} h_{0}}{t \sqrt{\pi}} \int_{0}^{t} \sqrt{t-\tau} d \tau=\frac{8 \lambda^{2} h_{0}}{3 \sqrt{\pi}} \sqrt{t} \\
W_{3}(t)= & -\frac{2 \lambda}{t \sqrt{\pi}} \int_{0}^{t} W_{2}(\tau) \sqrt{t-\tau} d \tau \\
= & -\frac{16 \lambda^{3} h_{0}}{3 t \pi} \int_{0}^{t} \sqrt{\tau} \sqrt{t-\tau} d \tau=-\frac{2 \lambda^{3} h_{0}}{3} t \\
W_{4}(t) & =-\frac{2 \lambda}{t \sqrt{\pi}} \int_{0}^{t} W_{3}(\tau) \sqrt{t-\tau} d \tau \\
& =\frac{4 \lambda^{4} h_{0}}{3 t \sqrt{\pi}} \int_{0}^{t} \tau \sqrt{t-\tau} d \tau=\frac{16 \lambda^{4} h_{0}}{45 \sqrt{\pi}} t^{3 / 2}
\end{aligned}
$$

taking into account that

$$
\begin{aligned}
\int_{0}^{t} \frac{\sqrt{t-\tau}}{\sqrt{\tau}} d \tau & =\frac{\pi}{2} t, & \int_{0}^{t} \sqrt{t-\tau} d \tau & =\frac{2}{3} t^{3 / 2} \\
\int_{0}^{t} \sqrt{\tau} \sqrt{t-\tau} d \tau & =\frac{\pi}{8} t^{2}, & \int_{0}^{t} \tau \sqrt{t-\tau} d \tau & =\frac{4}{15} t^{5 / 2}
\end{aligned}
$$


and their generalizations by

$$
\begin{gathered}
\int_{0}^{t} \tau^{n} \sqrt{t-\tau} d \tau=\frac{2^{n+1} n ! t^{n+\frac{3}{2}}}{(2 n+3) ! !}, \quad n \geq 1, \\
\int_{0}^{t} \tau^{n-\frac{1}{2}} \sqrt{t-\tau} d \tau=\frac{\pi(2 n-1) ! ! t^{n+1}}{2^{n+1}(n+1) !}, \quad n \geq 1 .
\end{gathered}
$$

The first step of the double induction principle is verified taking into account the above computations. For the second step, we suppose by induction hypothesis that we have (3.13) and (3.14). Therefore, we obtain

$$
\begin{aligned}
W_{2 n+2}(t) & =\frac{-2 \lambda}{t \sqrt{\pi}} \int_{0}^{t} W_{2 n+1}(\tau) \sqrt{t-\tau} d \tau \\
& =\frac{4 \lambda^{2} h_{0}}{t \sqrt{\pi}} \frac{\left(2 \lambda^{2}\right)^{n}}{(n+1)(n !)^{2}(2 n+1) ! !} \int_{0}^{t} \tau^{n} \sqrt{t-\tau} d \tau \\
& =\frac{2 h_{0}}{\sqrt{\pi t}} \frac{\left(4 \lambda^{2} t\right)^{n+1}}{(2 n+3)(n+1) ![(2 n+1) ! !]^{2}},
\end{aligned}
$$

and

$$
\begin{aligned}
W_{2 n+3}(t) & =\frac{-2 \lambda}{t \sqrt{\pi}} \int_{0}^{t} W_{2 n+2}(\tau) \sqrt{t-\tau} d \tau \\
& =-\frac{4 \lambda h_{0}}{t \pi} \frac{\left(4 \lambda^{2}\right)^{n+1}}{(2 n+3)(n+1) ![(2 n+1) ! !]^{2}} \int_{0}^{t} \tau^{n+\frac{1}{2}} \sqrt{t-\tau} d \tau \\
& =-2 \lambda h_{0} \frac{\left(2 \lambda^{2} t\right)^{n+1}}{(n+2)[(n+1) !]^{2}(2 n+3) ! !},
\end{aligned}
$$

by using 3.15 and 3.16 . Then, the proof by the induction principle holds.

Conclusion. We have obtained the global solution of a non-classical heat conduction problem in a semi- $n$-dimensional space, in which the source depends on the average of the total heat flux on the face $x=0$. Moreover, for the one-dimensional case we have obtained the explicit solution by using the Laplace transform and also the Adomian decomposition method.

\section{REFERENCES}

[1] G. Adomian, Solving frontier problems of physics: the decomposition method, Kluwer Academic Publishers, Dordrecht, 1994. MR 1282283

[2] G. Adomian, R. Rach, Analytic solution of nonlinear boundary value problems in several dimensions by decomposition, J. Math. Anal. Appl. 174 (1993), no. 1, 118-137. MR 1212921.

[3] G. Adomian, R. Rach, Modified decomposition solution of linear and nonlinear boundaryvalue problems, Nonlinear Anal. 23 (1994), no. 5, 615-619. MR 1297280

[4] L.R. Berrone, D.A. Tarzia, L.T. Villa, Asymptotic behavior of a non-classical heat conduction problem for a semi-infinite material, Math. Methods Appl. Sci. 23 (2000), no. 13, 1161-1177. MR 1779386 
[5] G. Bluman, P. Broadbridge, J.R. King, M.J. Ward, Similarity: generalizations, applications and open problems, J. Engrg. Math. 66 (2010), no. 1-3, 1-9. MR 2585810

[6] M. Boukrouche, D.A. Tarzia, Global solution to a non-classical heat problem in the semispace $\mathbb{R}^{+} \times \mathbb{R}^{n-1}$, Quart. Appl. Math. 72 (2014), no. 2, 347-361. MR 3186241

[7] M. Boukrouche, D.A. Tarzia, Non-classical heat conduction problem with nonlocal source, Bound. Value Probl. 2017, Paper No. 51, 14 pp. MR 3634826

[8] A.C. Briozzo, D.A. Tarzia, Existence and uniqueness for one-phase Stefan problems of nonclassical heat equations with temperature boundary condition at a fixed face, Electron. $J$. Differential Equations 2006, No. 21, 16 pp. MR 2198934

[9] A.C. Briozzo, D.A. Tarzia, Exact solutions for nonclassical Stefan problems, Int. J. Differ. Equ. 2010, Art. ID 868059, 19 pp. MR 2720039

[10] A.C. Briozzo, D.A. Tarzia, A Stefan problem for a non-classical heat equation with a convective condition, Appl. Math. Comput. 217 (2010), no. 8, 4051-4060. MR 2739647

[11] J.R. Cannon, The one-dimensional heat equation, Addison-Wesley, Reading, MA, 1984. MR 0747979

[12] J.R. Cannon, H.M. Yin, A class of non-linear non-classical parabolic equations, J. Differential Equations 79 (1989), no. 2, 266-288. MR 1000690

[13] H.S. Carslaw, C.J. Jaeger, Conduction of heat in solids, Clarendon Press, Oxford, 1959. MR 0959730

[14] A.N. Ceretani, D.A. Tarzia, L.T. Villa, Explicit solutions for a non-classical heat conduction problem for a semi-infinite strip with a non-uniform heat source, Bound. Value Probl. 2015, Paper No. 156, 26 pp. MR 3393801

[15] A. Friedman, Partial differential equations of parabolic type, Prentice Hall, Englewood Cliffs, N.J., 1964. MR 0181836

[16] K. Glashoff, J. Sprekels, An application of Glicksberg's theorem to set-valued integral equations arising in the theory of thermostats, SIAM J. Math. Anal. 12 (1981), no. 3, 477-486. MR 0613326

[17] K. Glashoff, J. Sprekels, The regulation of temperature by thermostats and set-valued integral equations, J. Integral Equations 4 (1982), no. 2, 95-112. MR 0654076

[18] N. Kenmochi, Heat conduction with a class of automatic heat source controls, In: Free boundary problems: theory and applications, Vol. II (Irsee, 1987), 471-474, Pitman Res. Notes Math. Ser., 186, Longman Sci. Tech., Harlow, 1990. MR 1081741

[19] N. Kenmochi, M. Primicerio, One-dimensional heat conduction with a class of automatic heat-source controls, IMA J. Appl. Math. 40 (1988), no. 3, 205-216. MR 0974674

[20] O.A. Ladyzenskaja, V.A. Solonnikov, N.N. Ural'ceva, Linear and quasilinear equations of parabolic type, Translations of Mathematical Monographs, Vol. 23, American Mathematical Society, Providence, R.I., 1968. MR 0241822

[21] R.K. Miller, Nonlinear Volterra integral equations, W.A. Benjamin, Menlo Park, Calif., 1971. MR 0511193

[22] N.N. Salva, D.A. Tarzia, L.T. Villa, An initial-boundary value problem for the onedimensional non-classical heat equation in a slab. Bound. Value Probl. 2011, Paper No. 4, 17 pp. MR 2821483

[23] D.A. Tarzia, L.T. Villa, Some nonlinear heat conduction problems for a semi-infinite strip with a non-uniform heat source, Rev. Un. Mat. Argentina 41 (1998), no. 1, 99-114. MR 1682205

[24] L.T. Villa, Problemas de control para una ecuación unidimensional no homogénea del calor, Rev. Un. Mat. Argentina 32 (1986), no. 3, 163-169. 
[25] A.M. Wazwaz, Linear and nonlinear integral equations. Methods and applications, Springer Heidelberg, 2011. MR 3024569

[26] A.M. Wazwaz, R. Rach, J.S. Duan, Adomian decomposition method for solving the Volterra integral form of the Lane-Emden equations with initial values and boundary conditions, Appl. Math. Comput. 219 (2013), no. 10, 5004-5019. MR 3009461

M. Boukrouche $e^{凶}$

Lyon University, UJM, Institute Camille Jordan, CNRS 5208, 23 Paul Michelon, 42023

Saint-Étienne Cedex 2, France

Mahdi.Boukrouche@univ-st-etienne.fr

D. A. Tarzia

Departamento de Matemática - CONICET, FCE, Universidad Austral, Paraguay 1950, S2000FZF Rosario, Argentina

DTarzia@austral.edu.ar

Received: December 24, 2018

Accepted: April 29, 2019 